\title{
Significant Improvement in The Piezoelectric Properties and Electromechanical Coupling Factors of Wurtzite AIN Compound Under High Pressures
}

\section{Z. A. A. R. Almaghbash}

Universite Dr Tahar Moulay de Saida

Arbouche Omar ( $\nabla$ arbouche_omar@yahoo.fr)

Universite Dr Tahar Moulay de Saida https://orcid.org/0000-0002-7406-5267

\section{A. Dahani}

Universite Dr Tahar Moulay de Saida

\section{A. Cherifi}

Universite Dr Tahar Moulay de Saida

\section{Belabbas}

Universite Dr Tahar Moulay de Saida

\section{B. Djellouli}

Universite Dr Tahar Moulay de Saida

\section{Research Article}

Keywords: Pressure; DFPT, piezoelectric properties, electromechanical coupling factors

Posted Date: February 15th, 2021

DOI: https://doi.org/10.21203/rs.3.rs-164626/v1

License: (1) This work is licensed under a Creative Commons Attribution 4.0 International License. Read Full License

Version of Record: A version of this preprint was published at Journal of Computational Electronics on September 13th, 2021. See the published version at https://doi.org/10.1007/s10825-021-01767-8. 


\title{
Significant improvement in the piezoelectric properties and electromechanical coupling factors of wurtzite AIN compound under high pressures
}

\author{
Z. A. A. R. Almaghbash ${ }^{1}$, O. Arbouche ${ }^{2,5}$, A.Dahani ${ }^{3,5}$, A.Cherifi ${ }^{1,5}$, M. Belabbas ${ }^{1}$ and \\ B. Djellouli ${ }^{4,5}$ \\ 1) Technology Laboratory of Communication, University of Saïda - Dr. Moulay Tahar. 20000 Saïda, Algeria \\ 2) Laboratory Physico-Chemistry of Advanced Materials, Djillali Liabes University of Sidi Bel-Abbes, 22000 \\ Sidi BelAbbes, Algeria \\ 3)Modelling and Simulation in Materials Science Laboratory, Djillali Liabès University of Sidi Bel-Abbès, \\ 22000, Sidi Bel Abbès, Algeria \\ 4) Laboratoire de Modélisation et Méthodes de calcul LMMC , University of Saïda - Dr. Moulay Tahar. 20000 \\ Saïda, Algeria \\ 5) Department of Electronics, Faculty of Technology, Dr. Tahar Moulay University of Saïda, 20000 Saïda, \\ Algeria
}

Corresponding author: arbouche_omar@yahoo.fr

zeyad.almaghbash@univ-saida.dz

\begin{abstract}
This work describes a theoretical study of the pressure effect in structural, elastic, piezoelectric and dielectric properties as well as electromechanical coupling factors of wurtzite AlN, obtained by ab-initio calculations using pseudo-potential plane waves (PP-PW) that combine the density functional theory (DFT) and density functional perturbation theory (DFPT). The results of the calculation indicate that the parameters of AlN crystal cells and the volume of AlN crystallin crystal cells decrease notably with increasing pressures from 0 to 40 $\mathrm{GPa}$. Due to an increase in the value of the direct piezoelectric constant ( $e_{33}$ and a decrease in the value of the elastic constant $\left(C_{33}\right)$, there is a significant improvement in the value of the converse piezoelectric constant $\left(d_{33}\right)$. The improvement in the piezoelectric value leads to a higher value in electromechanical coupling coefficient. Our results agree well with previous theoretical and experimental research. We hope that our results will provide guidelines for the realistic application as well as further research of high-performance compounds appropriate for applications in a multitude of fields of study, such as biomedical engineering.
\end{abstract}


Keywords: Pressure; DFPT; piezoelectric properties; electromechanical coupling factors

\section{Introduction}

Aluminum nitride (AlN) is one of the most important semiconductors used in various fields of applications such as optical electronic devices, cell phones, and optical detectors [1-3]. Given some important properties that this compound possesses, such as dielectric, mechanical, thermal conductivity and corrosion resistance. Owing to its $6.0 \mathrm{eV}$ (207 nm wavelength) large bandgap at ambient temperature, AlN can be used piezoelectric and ceramic with a wide variety of technical applications, such as ultrasound transducer [4,5]. The piezoelectric constants of AlN, however, are smaller than other widely used piezoelectric materials. For eg, piezoelectric strain modulus $d_{33}$ for $\mathrm{AlN}$ is reported to be $5.5 \mathrm{pC} / \mathrm{N}$, while $d_{33}$ for $\mathrm{ZnO}$ can be at least twice as large [6], and PZT can be larger than $100 \mathrm{pC} / \mathrm{N}$ [7]. Under normal temperature and pressure conditions, AlN compound crystallizes according to the hexagonal structure B4 (wurtzite), whose group space symmetry is of type P63mc or cubic structure (zinc blende) whose group space symmetry is of type F-43m (216 ) [8]. The change in structure under the influence of pressure causes an increase in internal energy which induces displacements of anions and cations, changes in chemical bonds, thus a change in the structure of energy bands. So studying the microscopic mechanism of phase transition is an essential ingredient. Despite this, there is some experimental work that has tried to explain some phase transition mechanisms [9-11]. The increase in pressure leads to an increase in the piezoelectric properties, which leads to an improvement in the electromechanical coupling factors. There is a crucial need to study materials and to know their physical properties, which include structural, elastic, piezoelectric and dielectric properties, in order to describe devices with extreme precision. A better understanding of structural properties provides a basic background for the production of engineering materials for advanced structures. The elastic and mechanical properties of solids reflect their reactions to the intervention of certain external factors. In the simplest case, such factors are mechanical actions: compression, traction, bending, shock, torsion. In addition to mechanical interventions, they can be thermal, magnetic, etc. Piezoelectric materials have many power applications. These materials have been used for many years, in applications such as ultrasonic generation or actuation. Recently, other applications have appeared, in particular with the piezoelectric transformer and microenergy sources. The transformer has found a commercial outlet in cold cathode lamp supplies for backlighting. Piezoelectric micro-sources, whose energy comes from ambient vibration, 
seem to have a bright future in applications such as the self-powering of abandoned sensor networks. In the present paper, we focus on the theoretical investigation of the piezoelectric and dielectric properties and electromechanical coupling factors of the wurtzite AlN compound at high pressure. The findings on structural, elastic, piezoelectric dielectric properties and electromechanical coupling factors under high pressure are described. The calculation have been reported after geometry optimization, as programmed in the open source software package ABINIT [12-14] within the framework of the DFT [15] and DFPT theories $[16,17]$. There are some previous computational studies in the influence of pressure on elastic properties [18-22]. Nevertheless, far as we know, there are no reports regarding the effect of pressure on piezoelectric, dielectric, and, electromechanical coupling parameters.

The rest of the paper is arranged on the basis of this. A brief overview of the methodology used and calculation description are given in Section 2. Section 3 presents and discusses the findings for structural, elastic, piezoelectric, dielectric and electromechanical coupling coefficients at high pressures. In section 4 , the findings are outlined.

\section{Theoretical methodology:}

First principles calculations within the framework of density functional perturbation theory (DFPT) have been shown to be the most reliable for obtaining elastic, piezoelectric and dielectric properties. This is the reason why we decided to use this method. The exchange and correlation potential is assessed using the local density approximation (LDA) based on the data of Ceperley and Alder [23] and generalized gradient approximation (GGA) using the scheme of the Perdew-Burke-Ernzerhof [24]. The calculations were conducted with the ABINIT code. The choice of this code was motivated by its open and recent development in the field of piezoelectric and dielectric properties. This code uses the pseudopotential approximation and plane waves as the basis for the development of Kohn-Sham orbitals. The pseudopotential used is nonlocal, norm conserving pseudopotentials (Pseudodojo) generated by Van Setten, M. J., et al. [25]. The potentials and the wave functions are represented in a plane wave base with a cut-off energy of $60 \mathrm{Ha}$. Integration into the Brillouin zone is performed in an 8x8x8 k-point mesh [26]. The elastic tensor with relaxed atoms results from the combination of the second derivative for electric field and strain;

$$
C_{\mathrm{ij}}=\frac{1}{\Omega_{0}} \frac{\partial^{2} E}{\partial \eta_{i} \partial \eta_{j}}
$$


where $\Omega_{0}$ is the cell volume, $\eta_{i}$ and $\eta_{j}$ the components of second-rank pure strain tensor. While the piezoelectric tensor with relaxed atoms takes into account the second derivatives for the internal stress, interatomic force constants, and effective Born charges. The proper piezoelectric constant can be obtained as:

$$
e_{\alpha \mathrm{j}}=\frac{\partial^{2} E}{\partial_{\alpha} \partial \varepsilon_{j}}+\mathrm{e}_{\alpha \mathrm{j}} \frac{1}{\Omega_{0}} Z_{\mathrm{m} \alpha}\left(K^{-1}\right)_{\mathrm{mn}} \Lambda_{\mathrm{nj}}
$$

Where, $\mathrm{Z}$ is the Born effective charges, $\varepsilon$ strain tensor. In addition, the dielectric tensor is described as:

$$
\epsilon_{\alpha \mathrm{j}}=1+4 \Pi \frac{\partial P_{\alpha}}{\partial E_{j}}
$$

Where $\mathrm{p}$ is the polarization induce by strain. All Those formulations are implemented in specific first-principles simulation packages ABINIT [12-14]. As well as the diagram below shows the full details for our calculation.

\section{Results and discussion}

\subsection{Structural properties}

The determination of structural properties in the physics of materials plays an important role, as it allows to gather information on the microscopic structure of materials and will therefore have a relatively significant effect on the prediction of other properties. The properties of the ground state of our materials are obtained using a relativistic scalar calculation based on the plane wave pseudo-potential (PW-PP) method, by treating the exchange and correlation energy by the LDA and GGA approximations, this choice is motivated by a concern for enriching the existing database; which will subsequently allow a comparative study of the performance of each of the approximations. In the frame 0-40 GPa, the optimum geometries of AlN unit cells have been calculated at constant values of applied pressure difference, where a full optimization for lattice parameter is carried out at each pressure. The result of optimization lattice constants a and c, the ratio c / a, the internal parameter $\mathrm{u}$ at zero-pressure of AlN wurtzite are indicated in Table1 with both LDA and GGA. Both the atomic positions and the lattice constants have been optimized in presence of hydrostatic pressure as and getting the pressures as function of volume to calculate the bulk modulus B and the pressure derivative of the bulk modulus B'. In addition, the empirical and previous theoretical results presented in the literature are also cited for comparison. In view of Table 1, we can notice that 
the value of a, c and $\mathrm{u}$ have been underestimated in light of the LDA with regard to the experiment [27], and vice versa, when we use the GGA, where it appears to be overestimated. In view of Table 1, lattice constants (a and c) and internal parameters obtained from LDA calculations are underestimated with regard to the experiment [27]. The study findings are satisfied with the actual results of the DFT-LDA and DFT-GGA methods [28,29]. In terms of theoretical calculations relative to those reported in [28] using all-electron full potential linearized augmented plane wave approaches, our LDA results in good consistency with the experiment [30]. For pressures varying between 0 and $40 \mathrm{GPa}$, our LDA values for a and c are compared to those of [31] and are shown to be lower. All parameters a, c, c/a and u are seen in Fig1. It should, however, be noted that the reported findings of our GGA agree well with those of [32]. Our LDA results are closer to the experiment for the bulk modulus at $\mathrm{P}=0$, and were found to be smaller by using the GGA approach rather than the LDA one. our values for $\mathrm{c}$ / $\mathrm{a}$ and $\mathrm{u}$ compared with the ideal structure ( $\mathrm{c} / \mathrm{a}=8 / 3$ and $\mathrm{u}=3 / 8$ ) [33], described in table 1 for both LDA and GGA approaches. In the current work, the computed $\mathrm{u}$ is in strong alignment with the experimental one described in [27]. Figure 1 indicates the pressure dependency of the ratio (c / a) and $\mathrm{u}$ for AlN, respectively. Notice that the $\mathrm{c} / \mathrm{a}$ ratio decreases as well for the compound of interest as pressure increases, whereas u increases.

\subsection{Elastic and related mechanical properties}

In the study of stability, elasticity is a very important factor, since it must be considered in the study of mechanical forces, it is a question of determining the elastic constants $C_{i j}$ which connect the stress to the strain and extract the mechanical and physical properties of these materials. The elastic constant results derived by the Abinit code shown in Table 2.It is acknowledged that wurtzite AlN has five distinct elastic constants, $C_{11}, C_{12}, C_{13}, C_{33}, C_{44}$. The elastic constants $C_{12}, C_{13}$ and, $C_{44}$ are linked to the form of elasticity. The elastic constants of the compound in question was determined in the current work for different compositions of pressure varying from 0 to $40 \mathrm{GPa}$ within both the LDA and GGA. In Table 2, our results are summarized and plotted in Fig 2. For comparison, the experimental findings and previous theoretical results reported in the literature are also shown. Our GGA for the elastic constants determined for w-AlN are in good accordance with the measured and determined data available from Ref [32]. and our LDA was found to be lower than [31] for pressure ranging from 0 to $40 \mathrm{GPa}$. The influence of hydrostatic pressure enables us to consider the mechanical stability and phase transition mechanisms of interatomic interactions. The $C_{i j}$ elastic constants follow the conditions of mechanical stability for w-AlN [34] in the 0-40 GPa pressure range, 
i.e. $C_{44}>0, C_{11}>C_{12} \vee, C_{33}\left(C_{11}+C_{12}\right)>C_{13}^{2}$ where, $C_{x x}=C_{x x}-P(x=1,2,3), C_{x y}=C_{x y}+P$, It shows that w-AlN for both LDA and GGA is stable in the hydrostatic pressure range described above. Certain physical quantities are strongly linked with the derived elastic constants, such as bulk modulus (B), shear modulus(G), etc.. If all the different elastic constants $C_{11}, C_{12}, C_{13}, C_{33}, C_{44}$ and $\left.C_{66}=1 / 2\left(C_{11}-C_{12}\right)\right)$ are extracted, it is possible to obtain the polycrystalline modulus using the Voigt Reuss methods [35-37]. Both bulk $\left(B_{V}, B_{R}\right)$, and shear modulus $\left(G_{V}, G_{R}\right)$ of AlN can be determined as :

$$
\begin{gathered}
B_{V}=\frac{1}{9}\left(2\left(C_{11}+C_{12}\right)+C_{33}+4 C_{13}\right), \\
B_{R}=\frac{\left(C_{11}+C_{12}\right) C_{33}-2 C_{13}^{2}}{C_{11}+C_{12}+2 C_{33}-4 C_{13}}(5) \\
G_{V}=\frac{1}{30}\left(C_{11}+C_{12}+2 C_{33}-4 C_{13}+12 C_{44}+12 C_{66}\right) \\
\left(C_{11}+C_{12}\right) C_{33}-2 C_{13}^{2}{ }^{2} C_{44} C_{66} \\
\left(C_{11}+C_{12}\right) C_{33}-2 C_{13}^{2}{ }^{2}\left(C_{44}+C_{66}\right) \\
3 B_{V} C_{44} C_{66}+ \\
G_{R}=\frac{5}{3}
\end{gathered}
$$

The bulk modulus (B) presented as follows: $B=1 / 2\left(B_{R}+B_{V}\right)$, and The shear modulus $(\mathrm{G})$ describes the plastic deformation resistance associated with the bending of the bonds depends on the nature of these bonds and decreases as a function of the ionicity: $G=1 / 2\left(G_{R}+G_{V}\right)$. For wurtzite structure, the shear anisotropies (A) are described as [38]: A shear = $2 C_{44} /\left(C_{11}-C_{12}\right)$. In addition, Young's modulus $(\mathrm{E})$, known as the stress-strain ratio, is generally used to calculate the rigidity of the solid, its defined as: $E=9 B G /(3 B+G)$. all those parameters $\mathrm{B}, \mathrm{G}$, and $\mathrm{E}$ with different pressures for $\mathrm{w}-\mathrm{AlN}$ are presented in Table 3 . The Poisson's ratio, against shear, calculates the stability of the crystal. The Poisson's ratio, which when it is between -1 and +0.5 , the material is said to be covalent with an ionic character. its defined by the following relationship: $v=(3 B-2 G) / 2(3 B+G)$. For $P=0$, $5,10,15,20,25,30,35$, and 40GPa, all described in Table 3. Although only those mechanical behaviors at ambient pressure have factual study for wurtzite AlN, our LDA and GGA calculations were first checked with published experimental results (Table3). The bulk B, shear G and Young modulus E experimental results are in good agreement with our GGA [31]. Under pressure, these major mechanical properties may be further studied. It has been found that B increases steadily with a corresponding increase in pressure (Table 3). Nevertheless, it has been found that with the rise in pressure (up to $5 \mathrm{GPa}$ in $\mathrm{G}$, and in E) with both LDA and GGA approximations, $\mathrm{G}$ and $\mathrm{E}$ modules gradually increase, then with more compression, the 
modulus B (up to $15 \mathrm{GPa}$ and $20 \mathrm{GPa}$ in G) with LDA and (25 GPa and 30 in E) with GGA essentially decrease, and eventually, we can notice that the elastic anisotropic A pressure rises from 0 to $40 \mathrm{GPa}$.

\subsection{Piezoelectric properties}

All non-centrosymmetric III-V compounds are piezoelectric, particularly nitrides (AlN, InN, and $\mathrm{GaN}$ ), this polarization is very important because the bond is strongly polarized, So if an external force is applied, the material deforms, the positions of atoms in relation to each other change, the parameters of the ideal mesh ? and ? and again change. Wurtzite AlN has three distinct piezoelectric tensor components, namely $e_{33}, e_{31}\left(=e_{32}\right)$, and $e_{15}\left(=e_{24}\right)$, which $e_{15}$ $\left(=e_{24}\right)$, and therefore can effectively be polarized along the z-direction as the system is strained in the same direction, i.e. the largest is the $e_{33}=\mathrm{xxx}$ constants. In order to determine the piezoelectric tensor in a wurtzite material, Both the atomic positions and the lattice constants have been optimized before beginning DFPT calculations. The calculated $e_{i j}$ are listed in table (4) and compared to previous data, which are only available for wurtzite at zero pressure $[39,40]$. Notice that our computed $e_{31}$ using the LDA is in close agreement with that recorded in [39] for wurtzite AlN. Moreover, the agreement between the determined $e_{15}$ of our LDA and that stated in[39] is fair. An even significant difference between our calculated $e_{33}$ LDA and that of [39] may, however, be noted. In addition, It can be inferred that our GGA results are in good accordance with the experiment [40], considering a well-known experimental uncertainty in the calculation of piezoelectric constants. Ueno, Masaki, et al. proved experimentally the phase transition from wurtzite-to-rocksalt at $22.9 \mathrm{GPa}$ [9]. Therefore, we decided to study The variation in the piezoelectric, dielectric properties and, electromechanical coupling coefficient versus pressure at $20 \mathrm{GPa}$ to ensure to ensure stability at this range. For e and d constants with LDA and GGA approximations, the range of the piezoelectric constants versus pressure is seen in table 4 and plotted in Fig3. Note that both piezoelectric constants $e_{31}$ and $e_{15}$ decrease as pressure increases, while $e_{33}$ increases. Same for converse piezoelectric constant $d_{33}$ increase whereas $\underset{(15)}{d}$ and $d_{31}$ decrease in both LDA, and GGA approximations. All piezoelectric constants have monotonic and nonlinear behavior. The obtained piezoelectric constant (d_33) of AlN system is 8.70, and $9.5 \mathrm{pC} / \mathrm{N}$, with GGA and LDA respectively which is $100 \%$ higher than that of the AIN at zero pressure. The 
improvement in the piezoelectric constants along z-direction are being related with internal parameter $\mathrm{u}$, so it depends on the $\mathrm{z}$-direction positions of $\mathrm{Al}$ and $\mathrm{N}$.

\subsection{Dielectric properties}

Dielectric constant describes the response of a given medium to an electric field. It is involved in many fields, in particular in optics, via the refractive index. The laws governing the refraction and reflection of light make use of it. At the microscopic level, the dielectric constant is linked to the electrical polarizability of the molecules or atoms constituting the medium. The dielectric constant is a tensor quantity (the response of the material can depend on the orientation of the crystallographic axes of the material), which reduces to a scalar in isotropic media. It is very generally complex; the imaginary part being linked to the phenomenon of absorption or emission of the electromagnetic field by the material. from (DFPT), we have calculated the Born effective charges $\left(Z^{B(k)}\right)$ and dielectric constants $(\varepsilon(\infty))$. Two separate components consist of the wurtzite system, one corresponding to the orientation parallel to the c-axis, $\varepsilon_{\perp}(\infty)=\varepsilon_{x x}(\infty)=\varepsilon_{y y}(\infty)$, and the other being perpendicular to the c-axis, $\varepsilon_{\|}(\infty)=\varepsilon_{z z}(\infty)$. Our findings with respect to $\varepsilon_{\perp}(\infty)$ and $\varepsilon_{\|}(\infty)$ for wurtzite AlN at $\mathrm{P}=0$ are found to be 4.35 and 4.57 for our LDA , 4.42 and 4.64 for GGA approximation. Which our GGA in good accordance with theoretical [41] The average value $\varepsilon(\infty)=(1 / 3) \operatorname{Tr} \varepsilon(\infty)$ at $\mathrm{P}=0$ has been calculated and found to be 4.43 and 4.52 for LDA and GGA respectively. which LDA good agree with available theoretical [41]. The variation of pressure versus $\varepsilon_{\perp}(\infty), \varepsilon_{\|}(\infty)$ and $\varepsilon(\infty)$ is shown in fig.4. Note that all the explored quantities decrease monotonically as pressure increases. Subsequently, the second derivatives of $\mathrm{E}$ are calculated with respect to the displacement of an atom and with respect to one of the components of $\varepsilon$, this forms the dynamic effective charge tensor (Born effective charge) $Z^{B(k)}$. Just one tensor of Born effective charges with two separate components should indeed be considered in the wurtzite structure. $Z_{\perp}^{B}=\left(Z^{B}\right)_{\mathrm{xx}}=\left(Z^{B}\right)_{\mathrm{yy}}$ and $Z_{\mathrm{II}}^{B}=\left(Z^{B}\right)_{\mathrm{zz}}$ [42]. Furthermore, the average value $Z^{B}$ Are being acquired as: $Z^{B}=(1 / 3) \operatorname{TrZ}^{B}$. Our determined showed the values of $Z_{\perp}^{B}$, $Z_{\mathrm{II}}^{B}$, and $Z^{B}$ for wurtzite $\mathrm{AlN}$ at $\mathrm{P}=0$ are 2.51, 2.66, and 2.51 with LDA and 2.51, 2.67, and 2.56 with GGA approximation. Our GGA value for $Z^{B}$ is in well accordance with the obtained value one of 2.57 reported in [43]. In addition, our GGA computed $Z_{\perp}^{B}$ and $Z_{\mathrm{II}}^{B}$ are in perfect accordance with [42] using DFT approach. In Fig.5, the pressure dependency of $Z_{\perp}^{B}, Z_{\mathrm{II}}^{B} \mathrm{k}$, and $Z^{B}$ for w-AlN is shown. Note that a pressure rises of up to $20 \mathrm{GPa}$ would not have a drastic impact on all quantities.

\subsection{Electromechanical coupling factor}

An interesting coefficient for the characterization of piezoelectric materials is the coupling coefficient (or factor) $\mathrm{k}$. It represents a material's ability to transform mechanical energy into electrical energy, and vice - versa. The coupling coefficient is defined for each mode of vibration, depending on the direction of polarization and the shape of the sample. These 
factors are essential parameters in the nature of the energetic harvesting and sensing transducer applications [44]. For applications including pressure sensors, ultrasound transducers and gyroscopes, the piezoelectric medium is usually needed to work in longitudinal mode [45], which the related coupling coefficient is:

$$
K_{33}^{2}=\frac{e_{33}^{2}}{\varepsilon_{33} C_{33}+e_{33}^{2}}(8)
$$

where $\varepsilon_{33}$ the dielectric tensor.

The high $K_{33}$ coupling factor contributes to higher axial resolution, broader bandwidth and transducers with greater sensitivity [46]. Cantilever-based actuators function in the mode of transverse length (bending) which the related coefficient of coupling is:

$$
K_{31}^{2}=\frac{e_{31}^{2}}{\varepsilon_{33} C_{11}+e_{31}^{2}}(9)
$$

Our values concerning $K_{33}$ and $K_{31}$ for $\mathrm{AlN}$ at $\mathrm{P}=0$ are found to be 0.29 , and 0.12 for our LDA, 0.26 and 0.12 with GGA approximation. Our GGA is well in accordance with theoretical [47] for $K_{33}$.and our LDA is well in consistent with the experimental value of 0.3 [48].The variation of the electromechanical coupling coefficients versus pressure are plotted in fig 6 . Note that both the $K_{33}$ and $K_{31}$ coupling coefficients increase as pressure increases. In addition, the factor K_33 reached a value of 0.38 and 0.40 with GGA and LDA respectively at $\mathrm{P}=20 \mathrm{GPa}$ which is comparable with $\mathrm{ZnO}$ [49], $\mathrm{BaTiO} 3$ [50], and, $\mathrm{LiNbO} 3$ [51] as candidate compound for practical considerations.

\section{Conclusion}

In summary, we have investigated the comprehensive effects of pressure on the structural, elastic, piezoelectric, dielectric properties, and electromechanical coupling coefficient of wurtzite AlN using calculations within density functional theory combined with density functional perturbation theory with both LDA, and GGA approximations. The pressure raises the piezoelectric response for the wurtzite phase considerably and the maximal piezoelectric coefficients that occur at the phase transition are much greater than those of the most typical ferroelectric perovskites. The improvement in the piezoelectric value leads to a higher value in electromechanical coupling coefficient. A promising direction for further theoretical studies can be useful for piezoelectric thin film in the future. 


\section{References}

[1] B. Monemar, Journal of Materials Science: Materials in Electronics 10.4 (1999) 227-254.

[2] Muralt, Paul Piezoelectric MEMS resonators. Springer, Cham, (2017) 3-37.

[3] Parks, D. A., Bernhard R. Tittmann, and M. M. Kropf. AIP Conference Proceedings. American Institute of Physics, 1(2010) 1211.

[4] Hou, Ruozhou, David Hutson, and Katherine J. Kirk. Insight-Non-Destructive Testing and Condition Monitoring, 55 (2013) 302-307.

[5] Kazari, Hanie, et al. IEEE Sensors Journal 19 (2019) 11090-11099.

[6] Kang, Xiaoyu, et al. Applied Physics Letters 110 (2017) 042903.

[7] Muralt, Paul, Journal of the American Ceramic Society 91 (2008)1385-1396.

[8] Barrigón, Enrique, et al. Chemical reviews 119 (2019) 9170-9220.

[9] Ojovan, Michael I., Lee, E. William Journal of Physics Condensed Matter 18 (2006) 11507-11520.

[10] Jaeger, Gregg Archive for History of Exact Sciences 53 199851-81.

[11] Imry, Yoseph, and Michael Wortis Physical Review B 19 (1979) 3580.

[12] Gonze, Xavier, et al. Computational Materials Science 25 (2002): 478-492.

[13] Gonze, Xavier, et al. Computer Physics Communications 180 (2009) 2582-2615.

[14] Gonze, Xavier, et al. Computer Physics Communications 205 (2016) 106-131.

[15] Sham, L. J. Philosophical Transactions of the Royal Society of London. Series A: Physical and Engineering Sciences 334 (1991) 481-490.

[16] Dal Corso, Andrea. Physical Review B 64 (2001) 235118.

[17] Gonze, Xavier, and Changyol Lee. Physical Review B 55 (1997) 10355.

[18] Ueno, Masaki, et al. Physical Review B 45 (1992) 10123.

[19] Wagner, J-M., and F. Bechstedt. Physical Review B 66.11 (2002) 115202. 
[20] Wright, A. F. Journal of Applied physics 82 (1997): 2833-2839.

[21] Saib, S., et al. Journal of Applied Physics 103 (2008) 013506.

[22] Li, Jing, et al. Applied Physics Letters 83 (2003) 5163-5165.

[23] Ceperley, David M., and Berni J. Alder. Physical Review Letters 45 (1980) 566.

[24] Perdew, John P., and Yue Wang. Physical review B 45 (1992) 13244.

[25] Van Setten, M. J., et al. Computer Physics Communications 226 (2018) 39-54.

[26] Monkhorst, Hendrik J., and James D. Pack. Physical review B 13.12 (1976): 5188.

[27] Ishihara, M., et al. Thin Solid Films 316.1-2 (1998): 152-157.

[28] Chen, Chang Peng, and Mei Lan Qi. Advanced Materials Research. Trans Tech Publications Ltd, 393 (2012).

[29] Wang, K., Xiao, Q., Xie, Q. et al. Journal of Elec Materi 48 (2019) 5135-5142.

[30] Schulz, Heinz, and K. H. Thiemann. Solid State Communications 23 (1977) 815-819.

[31] Zagorac, Dejan, et al. Theoretical and Applied Fracture Mechanics 103 (2019) 102289.

[32] Yong-Liang, Wang, et al. Communications in Theoretical Physics 49 (2008) 489.

[33] Saib, S., and N. Bouarissa The European Physical Journal B-Condensed Matter and Complex Systems 47 (2005) 379-383.

[34] G.V. Sin'ko and N.A. Smirnow, J. Phys. Condens. Matter. 14 (2002) 6989-7005.

[35] D.M. Teter,Computational alchemy MRS Bull. 23 (1998) 22-27.

[36] K. Tsubouchi and N. Mikoshiba, IEEE. Trans. Son. Ultrason. 32 (1985) 633-634.

[37] A. Reuss, Berechnung der Fliessgrenze von Mischkristallen auf Grund der Plastizitätsbedingung für Einkristalle, Math. Mech. 9 (1929) 49-58.

[38] K.B. Panda and K.S. Ravi Chandran, Computational Materials Science 35 (2006) 134150

[39] Momida, Hiroyoshi, Akihiko Teshigahara, and Tamio Oguchi. AIP Advances 6 (2016) 065006.

[40] Manna, Sukriti, et al. Physical Review Applied 9 (2018) 034026. 
[41] Saib, S., et al. Physica B: Condensed Matter 403 (2008) 4059-4062.

[42] Karch, Krystian, and Friedhelm Bechstedt. Physical Review B 56 (1997) 7404.

[43] Akasaki, I., and M. Hashimoto. Solid State Communications 5 (1967) 851-853.

[44] Wang, Qing-Ming, et al. IEEE transactions on ultrasonics, ferroelectrics, and frequency control 46.3 (1999) 638-646.

[45] Kim, Moojoon, Jungsoon Kim, and Wenwu Cao. Applied Physics Letters 87 (2005) 132901.

[46] Uchino, Kenji. Smart materials and structures 7 (1998) 273.

[47] Manna, Sukriti, et al. Journal of Applied Physics 122 (2017) 105101.

[48] Tadigadapa, S., and K. Mateti. Measurement Science and technology 20 (2009) 092001.

[49] Crisler, D. F., J. J. Cupal, and A. R. Moore. Proceedings of the IEEE 56 (1968) 225-226.

[50] Hong, Kuang-Sheng, et al. The Journal of Physical Chemistry Letters 1 (2010) 997-1002.

[51] Turner, R. C., et al. Applied acoustics 41.4 (1994) 299-324. 


\section{Figures captions}

Fig. 1. Dependence on the pressure of structural parameters (a) the lattice parameter a, (b) lattice constant c, (c) the ratio c/a, (d) the internal parameter $u$ of wurtzite AlN with LDA, and GGA approximations.

Fig.2. Dependence on the pressure of the elastic constants for wurtzite AlN with (a) LDA, and (b) GGA approximations.

Fig.3. Dependence on the pressure of the piezoelectric constants e $\left(\mathrm{C} / \mathrm{m}^{2}\right)$, and $\mathrm{d}(\mathrm{pC} / \mathrm{N})$ for wurtzite AlN with (a) piezoelectric constants e with LDA, (b) piezoelectric constants e with GGA approximations, (c) piezoelectric constants d with LDA, and piezoelectric constants d with GGA,

Fig. 4. Dielectric constant as function of pressure for AlN with (a) LDA, and (b) GGA approximations.

Fig.5. Born effective charge versus pressure for AlN with (a) LDA, and (b) GGA approximations.

Fig.6. Variation of electromechanical coupling parameters versus pressures for AlN with (a) LDA, and (b) GGA approximations. 
Figures
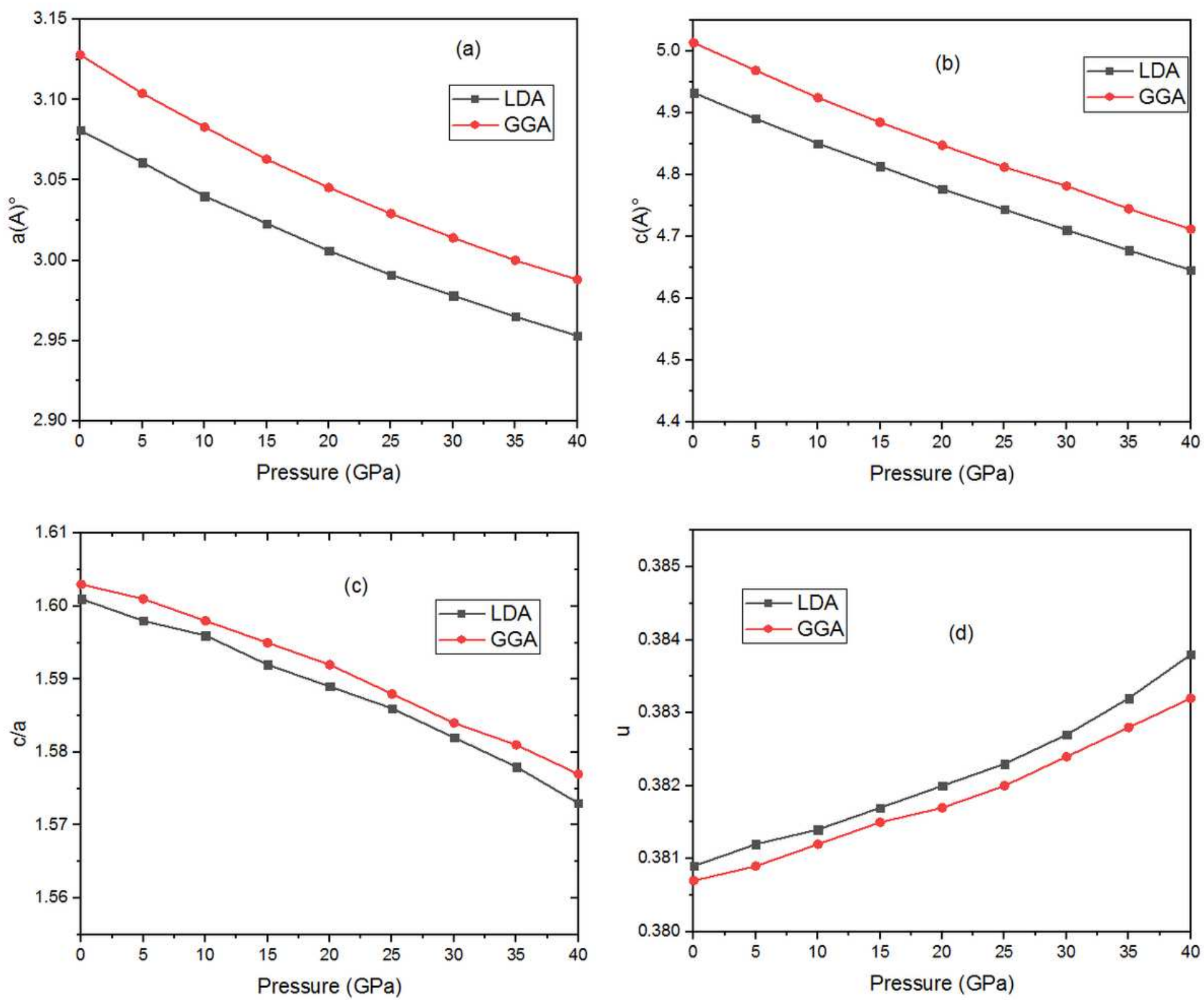

Figure 1

Dependence on the pressure of structural parameters (a) the lattice parameter a, (b) lattice constant c, (c) the ratio $c / a,(d)$ the internal parameter $u$ of wurtzite AIN with LDA, and GGA approximations. 

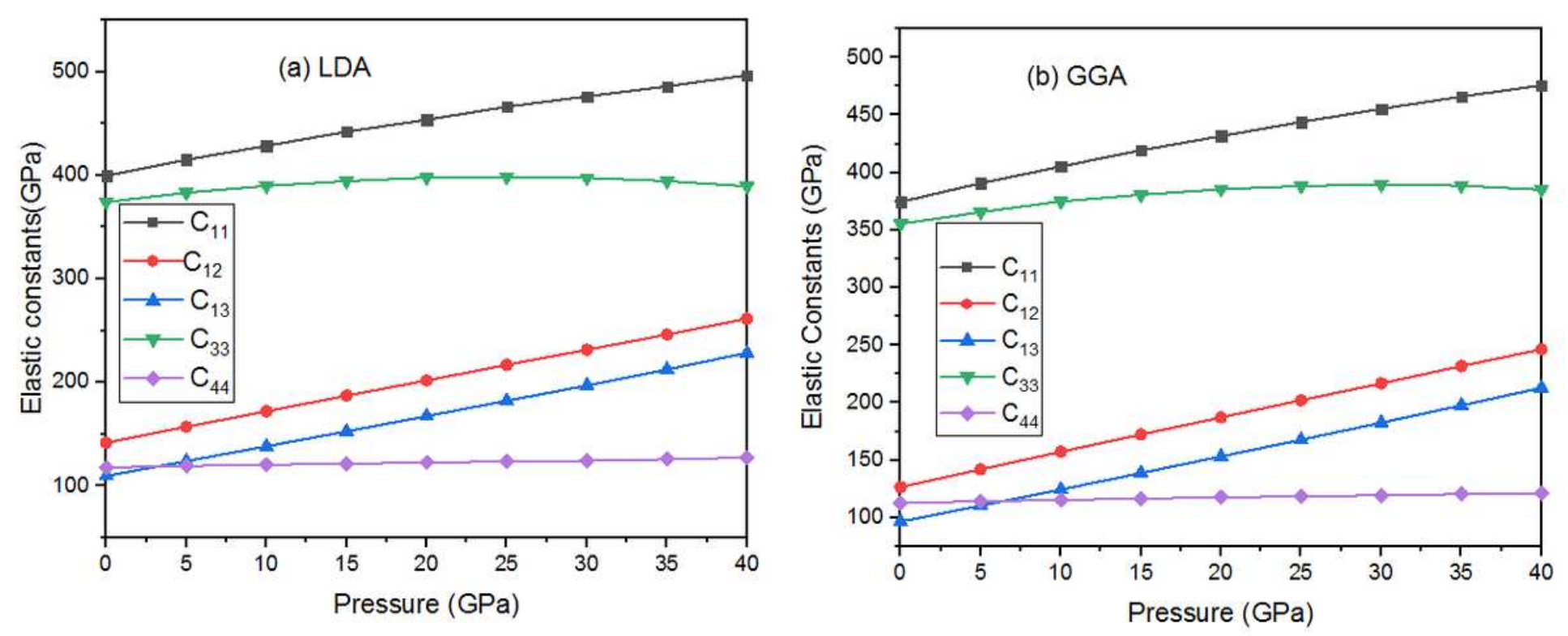

Figure 2

Dependence on the pressure of the elastic constants for wurtzite AIN with (a) LDA, and (b) GGA approximations. 

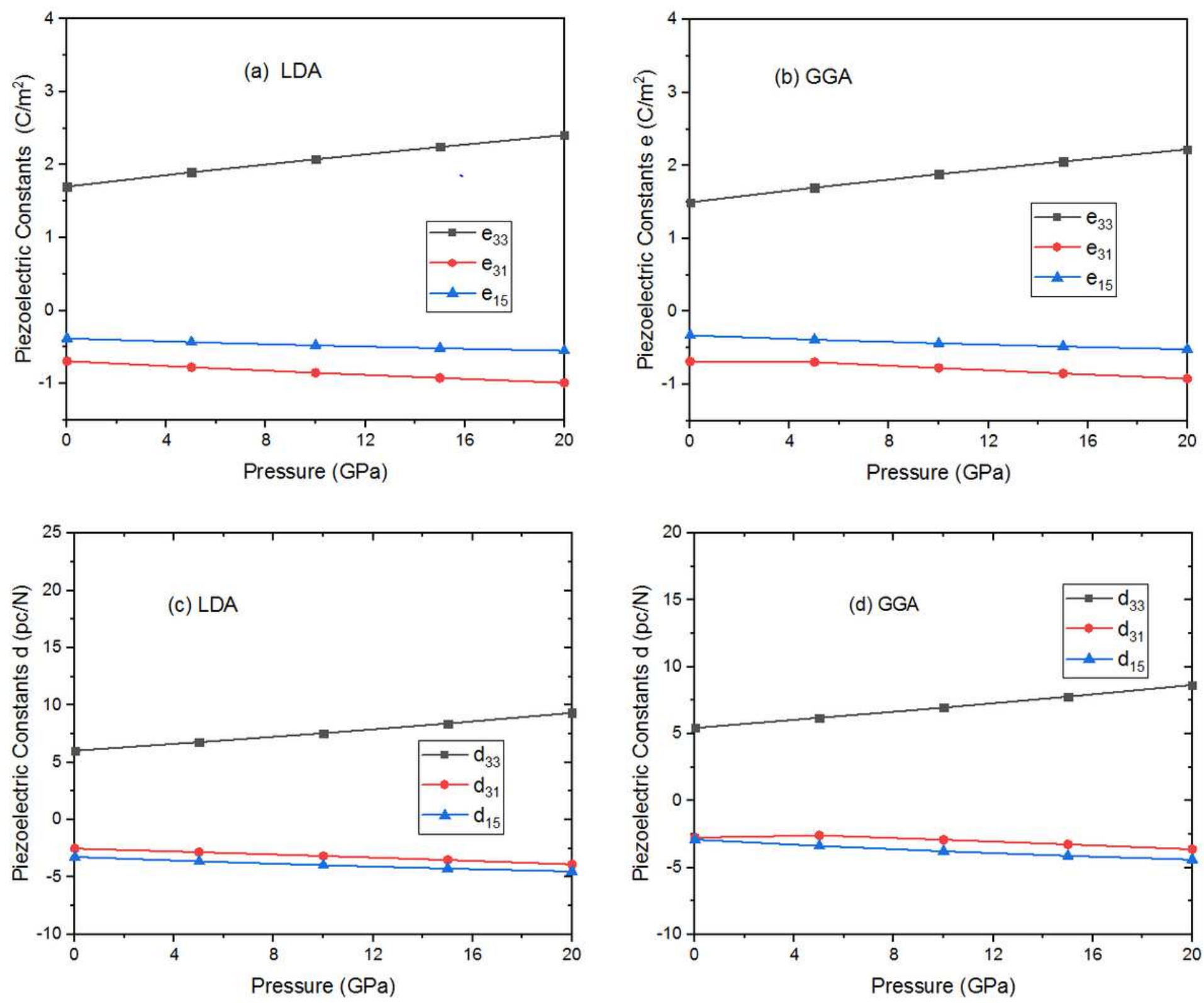

Figure 3

Dependence on the pressure of the piezoelectric constants e $(\mathrm{C} / \mathrm{m} 2)$, and $\mathrm{d}(\mathrm{pC} / \mathrm{N})$ for wurtzite AIN with (a) piezoelectric constants e with LDA, (b) piezoelectric constants e with GGA approximations, (c) piezoelectric constants $d$ with LDA, and piezoelectric constants $d$ with GGA, 

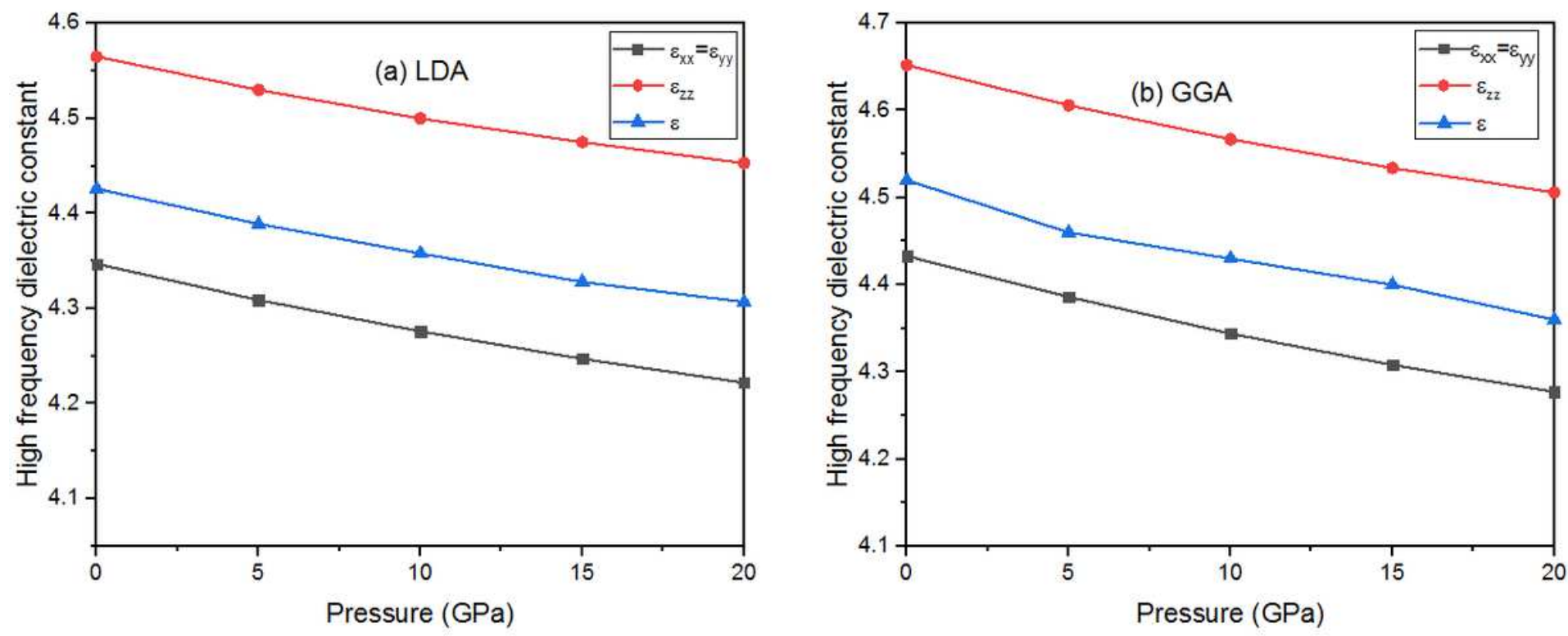

Figure 4

Dielectric constant as function of pressure for AIN with (a) LDA, and (b) GGA approximations.
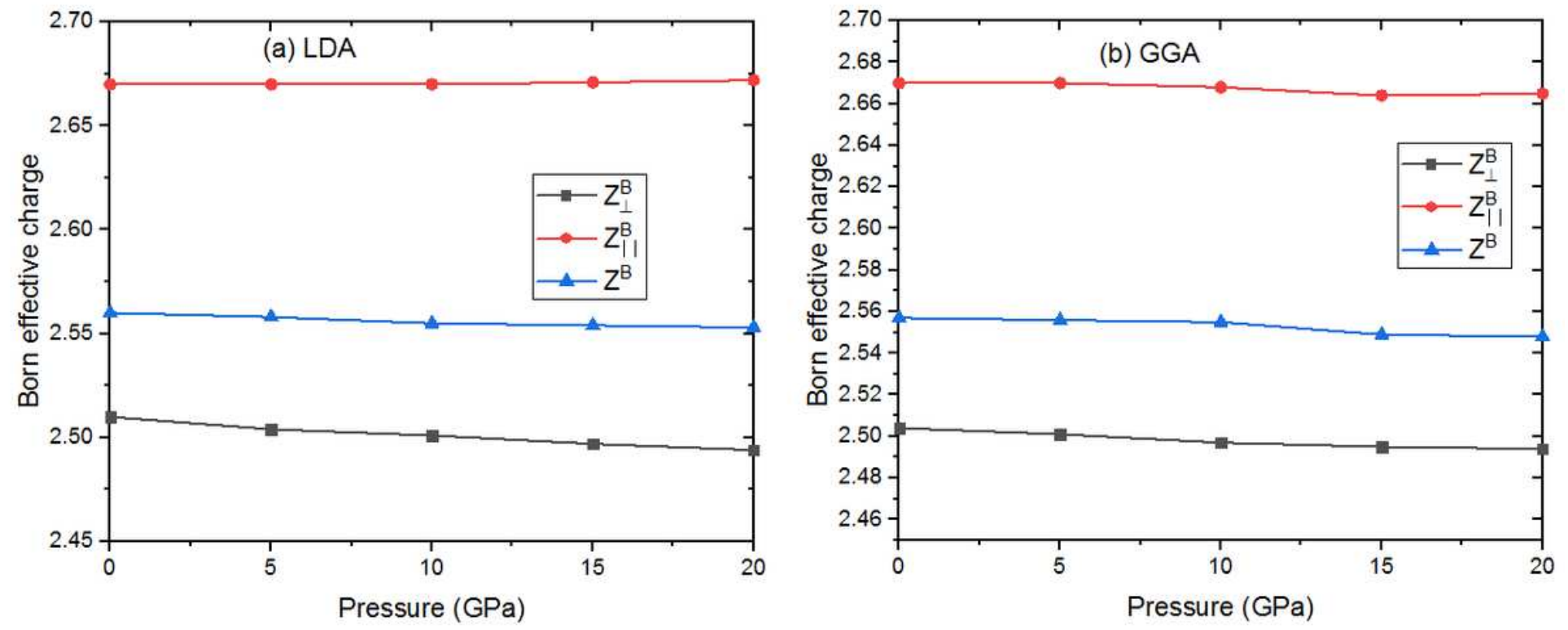

Figure 5

Born effective charge versus pressure for AIN with (a) LDA, and (b) GGA approximations. 


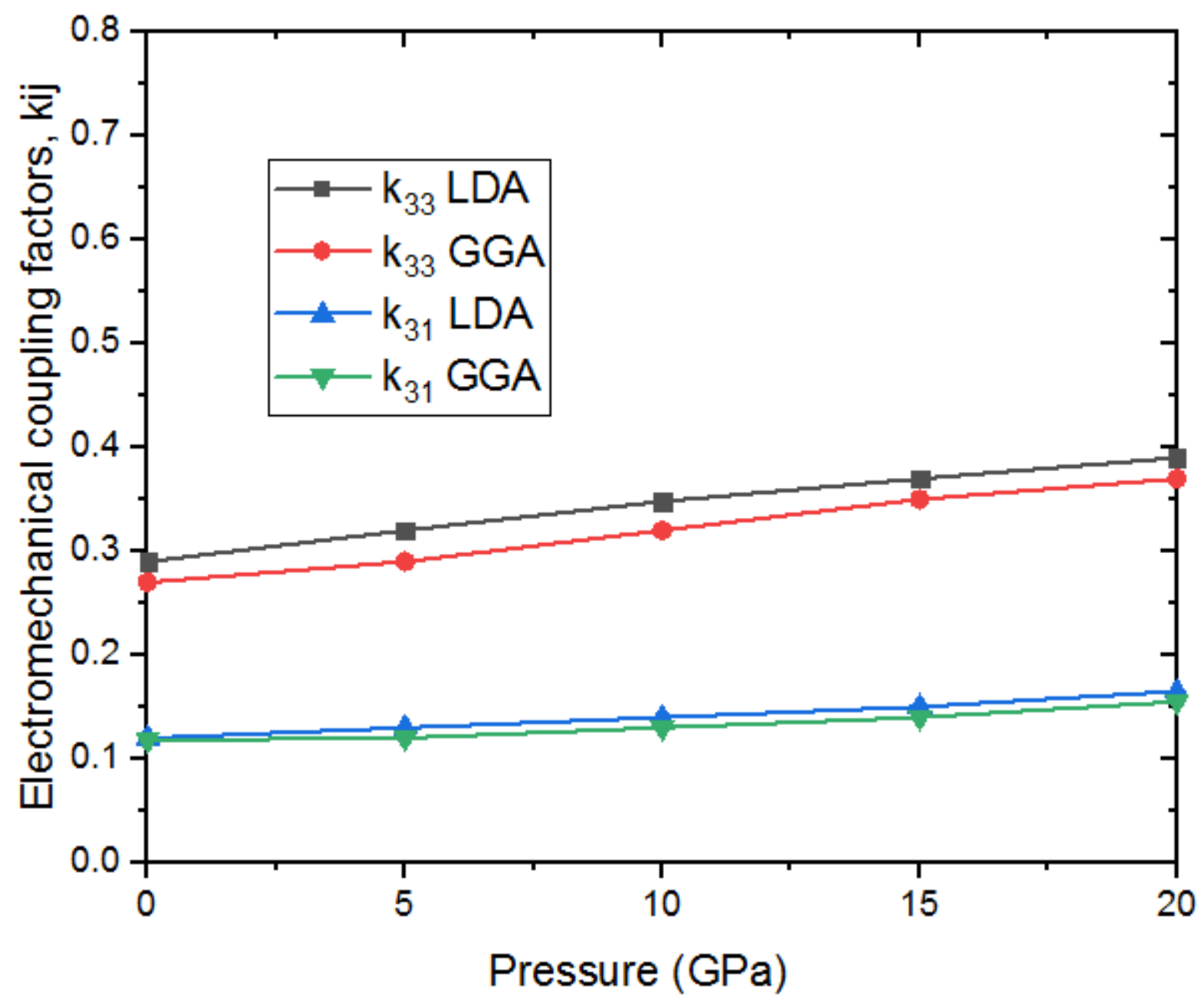

Figure 6

Variation of electromechanical coupling parameters versus pressures for AIN with (a) LDA, and (b) GGA approximations. 